https://doi.org/10.18485/efa.2019.11.ch24

\title{
Le Labyrinthe du monde de Marguerite Yourcenar comme texte performatif
}

\section{Nađa Đurić}

Faculté de Philologie, Université de Belgrade*

Ce travail propose de puiser dans les diverses tentatives d'appliquer les notions de « performatif » et d' " acte de langage » aux textes littéraires, dans le dessein d'expliquer la spécificité de la trilogie familiale de Yourcenar (Le Labyrinthe $d u$ monde : Souvenirs Pieux, 1974 ; Archives du Nord, 1977 ; Quoi ? L'Éternité, 1988, posthume). Dans ces « chroniques familiales et partiellement autobiographiques » (Yourcenar 1982), en alternant et fusionnant les genres, l'auteure brouille les limites entre écritures factionnelle et fictionnelle, pour élaborer une version de sa propre histoire familiale qui se substitue au vécu et acquiert la force d'événement. Le texte devient ainsi le lieu de construction et d'affirmation de son identité de fille et d'écrivaine.

Mots-clés : Marguerite Yourcenar, Le Labyrinthe du monde, performatif, actes de langage, genre, fiction.

\section{Introduction}

Le texte du Labyrinthe du monde, dernier ouvrage de Marguerite Yourcenar $^{1}$, est tissé si finement et avec une telle maîtrise, que le lecteur suivant les méandres de l'histoire familiale de Yourcenar ne s'apercevra peut-être pas qu'à la suite des époques et à l'enchevêtrement des destins évoqués correspond un réseau textuel tout aussi complexe et inextricable. L'écriture oscille entre autobiographique, mémorialiste, historiographique, essayiste et romanesque ; la critique yourcenarienne s'est beaucoup intéressée à cette hybridité générique, proposant des définitions telles que « hétérobiographie » (Maindron 1988), « roman historico-didactique » (Harris 1995) ou « histobiographie » (Park 2003). L'abondance

\footnotetext{
*nadja.djuric.fr@gmail.com

${ }^{1}$ Composé de trois volumes : Souvenirs Pieux, 1974 ; Archives du Nord, 1977 ; Quoi ? L'Éternité, posthume et inachevé, 1988.
} 
même des définitions suggère qu'il serait illusoire de rechercher une classification générique définitive du Labyrinthe du monde, et si nous nous intéressons à la complexité générique de ce texte, ce n'est pas pour proposer une définition de plus, mais pour essayer de déterminer l'enjeu d'une écriture qui embrasse les genres divers avec le plus grand naturel, et traite de manière équivoque le clivage entre littérature fictionnelle et non-fictionnelle.

C'est dans ce dessein que nous proposons d'interpréter la trilogie familiale de Yourcenar comme texte performatif. La notion de performatif a été introduite par J. L. Austin dans les conférences qu'il a prononcées à Harvard en 1955, publiées ensuite en 1962 sous le titre How to Do Things with Words (dans la traduction française : Quand dire, c'est faire, Austin 1970). Dans ce texte dont la réputation est désormais bien établie, Austin distingue deux types d'énoncés. Les énoncés constatifs décrivent la réalité, alors que les énoncés performatifs la produisent ; un énoncé performatif produit l'acte auquel il se réfère, comme se marier ou faire un pari. De ce fait, les performatifs ne sont pas vrais ou faux, mais heureux ou malheureux, ce qui dépend de l'issue de l'énonciation; pour qu'un performatif puisse être heureux, il doit exister une procédure reconnue, respectée par les participants et exécutée dans les circonstances adéquates. À partir de cette distinction, Austin procède à élaborer la théorie des actes de langage. S'inspirant des travaux d'Austin et de John R. Searle, dont le nom est également devenu indissociable de cette théorie, les linguistes ont poursuivi la réflexion sur le pouvoir performatif du langage.

En ce qui concerne une éventuelle application de cette approche théorique aux textes littéraires, la position d'Austin est claire et tranchée : il exclut la littérature du domaine de sa recherche, considérant qu'il « s'agit d'un usage parasitaire par rapport à l'usage normal » (Austin 1970 : 55). L'opinion de Searle est plus nuancée : il estime qu'il serait possible d'évoquer les actes de langage en littérature ; toutefois, puisque l'écrivain ne fait que prétendre de les accomplir, ils sont en fait des imitations d'actes que l'on peut accomplir dans la vie, et de ce fait dotés d'un statut inférieur (Searle 1982 : 101-119). Ces réserves initiales n'ont pourtant pas empêché une interrogation sur la possibilité d'appliquer cet outil théorique à l'analyse de textes littéraires, ni la constitution d'un corpus de textes sur les actes de langage en littérature.

Pour aborder de manière plus détaillée et systématique la manière dont la recherche littéraire s'est emparée de la théorie des actes de langage, on pourrait se référer à deux ouvrages de synthèse : le livre de Sandy Petrey, Speech Acts and Literary Theory, publié au Royaume-Uni (Petrey 1990), et celui de Kristina Peternai, Učinci književnosti: performativna koncepcija pripovjednog teksta, publié en Croatie (Peternai 2005²). Dans le cadre de cette étude, nous ne pourrons

\footnotetext{
${ }^{2}$ Souhaitant associer présentation et problématisation, Petrey a organisé son livre en trois parties : « Commencements », «Applications » et « Défis ». Dans l'ouvrage de Peternai, des chapitres sont
} 
proposer qu'un bref commentaire de cette problématique, entièrement plié aux nécessités de l'interprétation. Notre tâche sera d'examiner cette indécidabilité générique du Labyrinthe du monde dont nous venons d'esquisser les contours, pour la mettre ensuite en relation avec la performativité du texte littéraire, dans l'espoir que ce rapprochement nous permette de mieux saisir la spécificité de l'ouvrage de Yourcenar.

\section{Le labyrinthe de l'écriture}

Dans l'« Avant-propos de l'auteur » qui figure dans le volume de la collection de la Pléiade contenant ses œuvres romanesques, lorsqu'elle mentionne Le Labyrinthe du monde Yourcenar spécifie qu'il s'agit de « chroniques familiales et partiellement autobiographiques » (Yourcenar 1982 : IX). Cette désignation n'est certes pas incorrecte, mais elle s'avère insuffisante pour rendre compte de l'hybridité générique du texte, d'autant plus que l'auteure elle-même avait l'habitude de caractériser le troisième volume, Quoi ? L'Éternité, comme « mon roman » (Gaudin 1994 : 14). L'indécidabilité générique est confirmée par l'histoire de l'édition des trois volumes, qui ont paru d'abord successivement chez Gallimard, dans la collection Blanche où l'on publie en règle générale les romans ( « la grande collection de littérature et de critique françaises », précise-t-on sur le site internet de Gallimard), pour être ensuite publiés dans le second volume des œuvres de Yourcenar dans la Bibliothèque de la Pléiade, intitulé « Essais et Mémoires ».

L'incipit de l'ouvrage laisse entendre qu'il sera question de la vie de Yourcenar, ce qui autorise le lecteur à formuler un horizon d'attente conforme au genre de l'autobiographie :

L'être que j'appelle moi vint au monde un certain lundi 8 juin 1903, vers les 8 heures du matin, à Bruxelles, et naissait d'un Français appartenant à une vieille famille du Nord, et d'une Belge dont les ascendants avaient été durant quelques siècles établis à Liège, puis s'étaient fixés dans le Hainaut. (Yourcenar 1991 : 707)

La part autobiographique s'avère pourtant difficile à cerner, car loin de mettre l'accent sur « sa vie individuelle, en particulier sur l'histoire de sa personnalité » (Lejeune 1996 : 14), l'écrivaine diffère sans cesse le récit autobiographique proprement dit, pour le circonscrire finalement à quelques chapitres dans le troisième volume. Dans Souvenirs Pieux, Yourcenar raconte l'histoire de la famille maternelle ; le deuxième volume, Archives du Nord, est consacré à la lignée paternelle, mais le récit de la vie de son père est poursuivi dans le

consacrés à Austin et à Searle, mais également aux autres auteurs qui se sont donné pour tâche d'élargir le champ d'application de la notion de performatif : Jacques Derrida, Paul de Man, Mary Louise Pratt, Stanley Fish, Shoshana Felman, J. Hillis Miller et Judith Butler. 
troisième, Quoi ? L'Éternité, qui ne paraîtra qu'après la mort de l'auteure. Dans ce dernier volume, Yourcenar consent finalement à traiter son enfance dans un chapitre à part, et elle aborde également sa jeunesse, quoique de manière plus dispersée et fragmentaire. Le texte est resté inachevé, mais selon la « Note » d'Yvon Bernier, il ne manquait qu'une cinquantaine de pages « pour mettre un point final à l'entreprise » (Yourcenar 1991 : 1433); Bernier précise que l'auteure voulait encore raconter la mort de son père ainsi que ses propres débuts littéraires, et évoquer brièvement la période suivante de sa vie, jusqu'au début de la Seconde Guerre mondiale. Le récit autobiographique est ainsi doublement refusé : Yourcenar souligne dès le début la césure entre cet être qui lui est désormais étranger et son identité actuelle ( Que cet enfant soit moi, je n'en puis douter sans douter de tout », formule-t-elle ; Yourcenar 1991 : 707), et ne raconte son enfance et sa jeunesse que tardivement et de manière accessoire ; mais elle refuse également de raconter sa vie adulte et sa maturité littéraire.

Si la promesse autobiographique s'avère trompeuse, il paraît plus conséquent de lire les trois volumes du Labyrinthe du monde comme une chronique familiale. La stratégie d'écriture est déterminée par les sources mêmes dont l'écrivaine dispose. Ses ancêtres, propriétaires terriens, habitaient les mêmes régions depuis des générations, ce qui facilitait la conservation de documents et la transmission de l'héritage, et leur statut social favorisait l'acquisition d'un patrimoine. Ainsi l'écrivaine avait-elle accès à un matériel aussi riche que varié : sources d'archives et divers documents privés, portraits des ancêtres, sans oublier les témoignages oraux et les souvenirs personnels. Yourcenar construit son texte en travaillant des textes préexistants ; elle fait appel aux sources documentaires, incorpore des citations ou des paraphrases, et ce faisant elle tient à énoncer clairement sa manière de procéder. Pourtant, de même qu'elle invoque l'autobiographie pour ensuite la transcender, elle dépasse le cadre des chroniques familiales et fait preuve d'une ambition universalisante. «Ce qui m'intéressait, dans les deux livres [Souvenirs Pieux et Archives du Nord], c'était de remonter du presque présent au passé de la race tout entière », explique-t-elle à Matthieu Galey, ajoutant un peu plus tard : « je vois dans ce retour vers les millions d'êtres dont nous sommes faits la source d'une humilité très grande » (Yourcenar 1997 : 209, 211). L'histoire de la famille doit mener vers l'histoire de l'humanité toute entière.

En outre, la stratégie d'écriture varie en fonction des sources disponibles et de la distance - historique, temporelle et générationnelle, mais parfois aussi affective - qui sépare l'auteure de ses ancêtres. Le discours historiographique est utilisé surtout pour les périodes les plus lointaines ; lorsqu'elle ne dispose pas de sources textuelles, Yourcenar s'appuie sur les connaissances historiques. (Sun Ah Park met justement en relation l'approche utilisée dans Le Labyrinthe du monde avec la Nouvelle Histoire ; Park 2003). Le texte de Yourcenar admet ainsi une contradiction de plus, celle entre l'universalisme et l'historisme (Van 
der Starre 1995 : 426). Parallèlement à cette écriture documentaire, elle déploie une approche essayiste, présente surtout dans les nombreux commentaires dont le texte est parsemé. Lorsqu'elle veut traiter des sujets qui lui tiennent à cœur, s'opposer à la surpopulation, à la destruction de l'environnement, ou questionner les idées reçues sur l'enfance ou la maternité, Yourcenar n'hésite pas à se présenter comme une instance d'autorité détenant la vérité et imposant son avis au lecteur.

L'hybridité générique est davantage complexifiée par la différentiation entre écriture fictionnelle et non-fictionnelle. En réalité, cette distinction ne semble pas entraîner de difficultés dans les deux premiers volumes. Dans les "Carnets de notes de Mémoires d'Hadrien », Yourcenar dit travailler « [u]n pied dans l'érudition, l'autre dans la magie, ou plus exactement, et sans métaphore, dans cette magie sympathique qui consiste à se transporter en pensée à l'intérieur de quelqu'un » (Yourcenar 1982 : 526). Le même procédé est à l'œuvre dans sa trilogie familiale : lorsqu'elle possède suffisamment de données, Yourcenar transforme le document ou le témoignage par un traitement fictionnel. Seulement, elle prend soin d'attirer l'attention du lecteur aux éléments de fictionnalisation d'un texte censé être non-fictionnel. D'une part, elle cherche à contrôler l'apport de l'imagination en faisant recours, pour justifier ses hypothèses, aux explications basées sur la probabilité historique ou psychologique, la vraisemblance devant servir de garantie. De surcroît, elle n'omet pas de souligner le rôle de ses préférences personnelles dans la construction du vraisemblable (d'où l'emploi de formules telles que « J'aime à croire que » ou " J'imagine que »). D'autre part, elle cherche à rendre visible la part de fiction dans son texte : après avoir créée l'illusion fictionnelle, elle procède parfois à la détruire en exposant les rouages mêmes de son écriture.

Cependant, dans le troisième volume, et surtout lorsqu'elle s'intéresse aux époux de Reval qui n'appartiennent pas à la famille proprement dite, l'écrivaine renonce à ces cautions et abandonne l'ambition mémorialiste pour céder la place à la fantaisie romanesque. Cette partie du texte ressemble davantage à un roman ; le lecteur peut s'apercevoir que la liberté d'écriture est plus grande, l'invention plus importante, et que le commentaire auctorial visant à délimiter la juste place de l'imagination se fait de plus en plus rare. En fait, Yourcenar avait d'abord conçu son ouvrage comme un diptyque ; la structure tripartite s'est imposée en cours de route, et en même temps l'écriture a évolué de façon spontanée de la non-fiction vers la fiction.

Le besoin d'aborder son histoire familiale de cette manière s'explique par l'enjeu même de cette histoire. Au centre du labyrinthe familial, réitèrent les critiques, se trouve la mère reniée et rejetée, Fernande de Crayencour, morte peu après l'accouchement d'une fièvre puerpérale, ce qui aurait provoqué chez l'écrivaine une culpabilité inconsciente et une dévalorisation généralisée de la maternité. Si la vie de Fernande est dûment traitée, la jeune femme est systématiquement 
présentée comme insuffisante, malgré certaines qualités qui lui sont accordées. Dans le troisième volume, en revanche, Yourcenar se penche sur la vie de Jeanne de Reval, amie de pension de sa mère et amie, peut-être aussi amante, de son père : à la différence de la mère biologique, cette mère symbolique devient une figure idéalisée qui sert de modèle à Yourcenar. Mais il ne suffit pas à l'écrivaine de faire le récit de la vie de Jeanne (une vie que d'ailleurs elle n'a pas les moyens de connaître de manière aussi intime). Sa démarche est autre : sans l'énoncer clairement, Yourcenar transmet au personnage de Jeanne sa propre expérience, et s'inspire de sa propre vie pour imaginer celle de Jeanne de Reval ${ }^{3}$. En projetant ainsi son expérience personnelle, elle crée une transmission et une proximité psychologique qui sont en réalité le produit de son désir.

Une fois que la narration a commencé à obéir à la logique du désir, il semble qu'il ne soit plus possible de revenir en arrière : de l'histoire de Jeanne, mère rêvée et désirée, Yourcenar passe à l'histoire du mari de Jeanne, Egon de Reval. Dans le dernier chapitre rédigé, «Les Sentiers enchevêtrés », Egon, qui est d'origine balte, retourne dans son pays déchiré par la guerre pour retrouver sa famille. Il n'est plus question d'histoire familiale : en fait, Yourcenar introduit dans ses mémoires le décor et l'ambiance de son récit Le Coup de grâce (Proust 2001 : 121-129). L'ambition documentaire est clairement abandonnée au profit du traitement romanesque. Egon pourrait même être perçu comme un alter ego de l'écrivaine : sa rencontre avec le corps décrépit d'une mère incapable de le reconnaître permet à Yourcenar de se confronter de manière symbolique à l'image de la mère reniée. Simone Proust l'interprète come une confirmation du dégoût de Yourcenar pour la procréation (ibidem : 128), mais le fait de mettre ainsi en fiction sa propre fascination, et de plus dans un épisode qui devait précéder la clôture de ses mémoires, suggère au moins la volonté de faire face au contenu réprimé.

En confondant les récits autobiographique et biographique, en introduisant l'écho de son œuvre fictionnelle dans son histoire familiale, Yourcenar arrive à brouiller les frontières entre sa vie et celle des autres, et finalement entre sa vie et son œuvre. Ainsi la porosité entre les genres divers lui permet d'écrire une histoire familiale au statut ambivalent. Elle met en place une version de son histoire familiale qu'elle choisit pour sienne, et en la fixant par le texte, elle la pose comme authentique et définitive.

\section{Les actes de langage et la théorie littéraire}

Pour déterminer en quoi consiste la performativité dans Le Labyrinthe $d u$ monde, il serait judicieux de revenir sur l'emploi de la notion de l'acte de langage

\footnotetext{
${ }^{3}$ Particulièrement lorsqu'il est question de sa relation avec Jerry Wilson (voir à ce sujet la biographie de Yourcenar par Josyane Savigneau, Savigneau 1990 : 595-664).
} 
en théorie littéraire. Force est de constater qu'il n'y a pas d'unanimité à ce sujet, bien que Thomas Pavel ait raison de souligner la récurrence d'« une structure assez caractéristique, que l'on retrouve dans d'autres applications de modèles formels aux phénomènes littéraires » et qu'il nomme « modèle en panne » (Pavel 1988 : 34-35) : « on affirme d'abord que la littérature forme une sous-classe d'un champ plus vaste ; on présente ensuite un modèle de ce champ ; on démontre enfin que les phénomènes littéraires ne peuvent pas être saisis à partir des distinctions offertes par le modèle proposé » (ibidem : 35).

En premier lieu, le niveau où l'on situe l'acte de langage peut varier. Toutefois, cette indétermination était déjà présente dans les conférences d'Austin, et constitue un enjeu important de sa réflexion. En fait, Austin ne parvient pas à maintenir la distinction nette entre les constatifs et les performatifs qu'il avait d'abord établie. Il est amené à distinguer trois types d'actes : locutoire (production de sons et de mots douée d'une signification), illocutoire (la manière dont on utilise le discours) et perlocutoire (effet produit par le discours) (Austin 1970 : 109-118). Il formule ainsi le rapport entre la locution et l'illocution : la locution est « l'acte de dire quelque chose », et l'illocution « un acte effectué en disant quelque chose » (ibidem : 113). Puisque l'acte illocutoire n'est pas séparable de la locution, on arrive à une extension du pouvoir d'agir du discours, ainsi qu'à une nouvelle classification des énonciations en fonction de leur valeur illocutoire : verdictifs, exercitifs, promissifs, comportatifs et expositifs (ibidem : 153).

Le même vacillement entre les conceptions restreinte et généralisée de la performativité est présent dans la théorie littéraire. À l'instar de la démarcation entre les performatifs explicites et la force illocutoire inhérente à tout discours, le texte littéraire peut être perçu comme comprenant des actes de langage, ou comme provenant d'un acte de langage lui-même. Assez souvent, les deux possibilités sont considérées, et il s'agit d'examiner leur rapport.

Certains auteurs soulignent l'imbrication des deux niveaux et envisagent le texte de fiction comme composé d'autant d'énoncés qui possèdent une force illocutoire (même si elle est considérée comme simulée, dans la logique du «modèle en panne » évoqué ci-avant). Telle est la conclusion de Richard Ohmann, qui écrit : «Une œuvre littéraire est un discours dont les phrases manquent de forces illocutoires qui leur seraient normalement attachées. Leur force illocutoire est mimétique» (Ohmann 1971 : 14 ; nous traduisons). Tel est le jugement de Searle, qui affirme que " l'auteur d'une œuvre de fiction feint d'accomplir une série d'actes illocutoires, notamment du type assertif» (Searle $1982: 108$ ). Et lorsque Gérard Genette, dans Fiction et diction, entreprend de compléter la position de Searle, il explique qu'en produisant des assertions feintes, l'auteur accomplit en même temps un autre acte, qui est la création d'une œuvre de fiction (Genette 2004 : 125-126), ce qui l'amène à définir les énoncés de fiction comme « des assertions feintes recouvrant, de manière plus ou moins évidente et transparente, 
des déclarations (ou demandes) tout à fait sérieuses que l'on doit tenir pour des actes illocutoires » (ibidem : 135).

Dans certains textes, on maintient la co-présence des deux niveaux, mais une perspective différente est introduite. Les auteurs ne traitent pas tous les énoncés qui forment un texte de fiction comme possédant une force illocutoire, mais se concentrent sur les textes qui thématisent la performativité du langage et comprennent des performatifs spécifiques. Ainsi Stanley Fish, dans son étude de Coriolan, analyse la tragédie de Shakespeare comme un texte qui traite particulièrement des actes de langage, et dans la partie théorique de son étude il aboutit à la conclusion que la théorie des actes de langage ne pourrait pas constituer une approche littéraire, sauf dans les cas bien précis où le texte interprété le justifie (Fish 1980). D'autres auteurs choisissent également d'analyser des textes précis, ou des corpus de textes qui se prêtent à ce type d'analyse, mais à la différence de Fish, en même temps qu'ils analysent des actes de langages thématisés ou incorporés dans les textes, ils élaborent des conceptions spécifiques de la performativité généralisée du texte littéraire. Ainsi Shoshana Felman interprète Don Juan de Molière en insistant sur la promesse subvertie et la rhétorique de séduction utilisée par le personnage éponyme, pour analyser ensuite le « donjuanisme d'Austin » et mettre en relation la substitution du critère de satisfaction au critère de vérité avec la manière dont la psychanalyse de Lacan aborde le rapport entre le langage et le référent (Felman 1980). J. Hillis Miller, qui a écrit deux ouvrages sur le sujet, se propose d'explorer la diversité des actes de langage dans les romans de Henry James (Miller 2005), mais il conclut son premier livre, essentiellement théorique, par l'analyse de quelques épisodes du roman $\dot{A}$ la recherche du temps perdu de Proust où la performativité n'est pas dans les actes de langage isolés et définissables, mais dans la création performative de l'autre dont la vérité demeure insaisissable (Miller 2001). Dans un autre ouvrage encore plus récent, Mario Ortiz Robles étudie le roman victorien où les actes de langage tels que se marier ou léguer/hériter propulsent l'intrigue (paradoxalement, surtout lorsqu'ils sont malheureux) et contribuent à la construction discursive de la réalité, mais son interprétation est ancrée dans les réflexions sur la capacité du genre romanesque à modéliser la formation du sujet en insistant sur sa performativité (Ortiz Robles 2010).

Il s'agit en fait d'un autre type de généralisation de la performativité, auquel fait référence J. H. Miller lorsqu'il distingue deux manières d'hériter d'Austin. Selon la première, représentée surtout par Searle, l'acte de langage dépend d'un sujet conscient, en pleine possession de ses pouvoirs ; puisque cet acte est intentionné et dépend des règles, procédures et conventions, il est possible d'envisager une théorie cohérente des actes de langage. Pour la seconde, défendue par les représentants de la déconstruction (Derrida, Felman, Butler, etc.) une telle théorie est inconcevable ; le performatif désigne le pouvoir des mots en général, et ce 
pouvoir est indépendant du sujet parlant ou écrivant (Miller $2005: 5-6,8$ ). Nous avons déjà précisé la position de Felman. Quant à Derrida, dont la polémique avec Searle est bien connue, il s'oppose à la conception austinienne du performatif en insistant sur son itérabilité et sur l'impossibilité d'en saturer et contrôler le contexte ; il s'attaque ainsi à l'idée d'une source d'énonciation comme affirmation du logocentrisme (Derrida 1990). Butler s'est beaucoup intéressée à la performativité du genre, mais aussi aux implications éthiques et politiques de la performativité du langage. Pour ces auteurs, la réflexion sur le pouvoir des mots (titre d'un ouvrage de Butler), s'articule à la conception du sujet comme étant en construction perpétuelle, à l'opposé de l'idée d'une identité stable autrefois présupposée. Dans cette optique, la performativité semble se dissoudre dans la textualité et se confondre avec la conception même du sujet ${ }^{4}$.

\section{La performativité du Labyrinthe du monde}

Il est clair que dans le cas de la trilogie familiale de Yourcenar une conception généralisée de la performativité ne saurait rendre compte de la spécificité du texte (même s'il nous faudra revenir sur la question de la construction du sujet) ; mais il n'est pas question non plus d'un texte thématisant des actes de langage.

Reprenons la terminologie de Searle, qui nous sera utile par la suite. Sa taxinomie des actes illocutoires comprend entre autres les assertifs, " dont le but est d'engager la responsabilité du locuteur (à des degrés divers) sur l'existence d'un état des choses, sur la vérité de la proposition exprimée » (Searle 1982 : 52 ) et les déclarations, dont « l'accomplissement réussi garantit que le contenu propositionnel corresponde au monde » (ibidem : 57). Une catégorie particulière des déclarations est appelée « déclarations assertives » : il est question des déclarations prononcées dans certaines situations institutionnelles où « nous recourons à une autorité pour statuer sur ce que sont les faits », et de ce fait le jugement ou l'évaluation acquièrent la force de déclaration, p. ex. "Vous êtes coupable » si prononcé par un juge (ibidem : 59).

Le texte de Yourcenar, i.e. les parties du texte à forte référentialité, correspondant à la définition de la chronique, biographie ou autobiographie, sont basées sur la distinction du vrai et du faux, et peuvent être traitées comme des assertifs. Les chapitres orientés vers la fictionnalisation se présentent comme des assertifs, alors que l'acte illocutoire accompli est en effet la déclaration (c'est au fond le modèle proposé par Genette dans Fiction et diction).

\footnotetext{
${ }^{4}$ Cette manière de généraliser la performativité est justement illustrée par l'ouvrage déjà mentionné de Kristina Peternai (Peternai 2005). Dans la deuxième partie de son livre, Peternai consacre un chapitre à la performativité du texte narratif ; elle s'appuie sur les théories structuralistes et narratologiques pour traiter de la construction de l'identité et de la question du sujet (auteur ou lecteur).
} 
Si nous mettons en relation performativité et fictionnalisation, il nous faut d'abord interroger et confirmer le caractère fictionnel du texte en question. Dans certaines parties du troisième volume, celui que Yourcenar désigne par le terme de roman, nous retrouvons les deux propriétés spécifiques de la fiction soulignées par Dorrit Cohn dans Le propre de la fiction : « (1) ses références au monde extérieur au texte ne sont pas soumises aux critères d'exactitude ; et (2) elle ne se réfère pas exclusivement au monde réel, extérieur au texte » (Cohn $2001: 31$ ). La première est plus évidente dans le chapitre sur le voyage d'Egon de Reval que dans les chapitres sur Jeanne, bien qu'une lecture attentive de ces séquences et leur comparaison avec les biographies de l'auteure ne laissent pas de place à l'incertitude. La deuxième porte sur la manière de représenter la vie intérieure, permettant au narrateur de « connaître des choses qu'on ne saurait connaître ni dans le monde réel ni dans les fictions ayant comme finalité une représentation du monde réel » (ibidem : 33). Conformément à l'analyse de Cohn, la vie intérieure de ces personnages est présentée avec une transparence que seule la fiction peut accorder. Il faut bien rappeler que c'est également le cas, le plus souvent, des chapitres à l'ambition référentielle, mais il s'agit alors d'une utilisation ponctuelle, ou partielle, ou exhibée comme procédé de fictionnalisation et justifiée par le maniement de sources textuelles, de témoignages ou souvenirs.

Pourtant, cette délimitation entre deux régimes d'écriture s'avère particulièrement délicate dans la mesure où dans le régime dit fictionnel l'énonciateur est toujours le même, et toujours réel, ce qui devrait réfuter l'hypothèse sur le caractère fictionnel de certaines parties du texte. Le récit de la vie de Jeanne de Reval en est le meilleur exemple. Les chapitres qui lui sont consacrées ne sauraient être rapprochés aux cas recensés par Dorrit Cohn : il ne s'agit pas d'une biographie fictionnelle, car la personne dont on raconte la vie n'est pas imaginaire. Certes, dans le $5^{\mathrm{e}}$ chapitre, où elle étudie Sir Andrew Marbot, ouvrage de Wolfgang Hildesheimer, Cohn met en évidence l'existence d'un « courant expérimental postmoderne : le récit de vie qui se situe à (et joue sur) la frontière entre l'histoire et la fiction » (ibidem : 146), mais elle donne l'exemple de l'effondrement de la distinction générique entre autobiographie historique et fictionnelle, i.e. de l'autofiction. (Dans l'autofiction, comme le rappelle Schaeffer, « l'auteur réel, identifié par son nom d'auteur, assume un récit fictif» (Schaeffer 1989: 85), mais chez Yourcenar les séquences dont la véracité et la référentialité s'avèrent problématiques ne concernent pas l'auteure, mais Jeanne et Egon de Reval). Pour ce qui est de « la fiction et l'histoire dans le champ des récits narrant la vie d'un autre », Cohn constate que « la transgression expérimentale ne s'est exercée que dans une direction », dans les textes qui « se servent des procédés fictionnels [...] pour rendre compte de la vie intérieure de personnes réelles - actrices de cinéma, meurtriers reconnus coupables, astronautes, héros sportifs » (Cohn 2001 : 146-147). Mais il serait encore difficile de placer le texte de Yourcenar dans 
cette catégorie, et ce pour plusieurs raisons. À la différence de ces ouvrages qui énoncent clairement leur manière de procéder par des sous-titres oxymoriques ( «oman véridique », « Biographie roman », «Roman de non fiction»; ibidem) la nature du texte de Yourcenar n'est pas explicitement désignée ; de plus, come nous l'avons déjà montré, il est assimilé à un texte référentiel des plus traditionnels. Aussi, la personne dont on raconte la vie n'est pas connue du public, ce qui est important dans la mesure où une telle connaissance contraindrait au respect du critère de la vérité, et servirait à la fois de mise en garde contre les procédés de fictionnalisation.

Si la distinction entre fiction et non-fiction est brouillée, elle l'est pour une raison primordiale. Selon E. D. Hirsch, « la finalité d'un genre est, ni plus ni moins, la finalité communicationnelle que poursuit un locuteur particulier » (cité dans Schaeffer 1989: 74) et pour Yourcenar, cette finalité est double : décrire le monde, de la famille à l'humanité, et en même temps élaborer un roman familial dans le sens ou l'entend Marthe Robert (dans le cas de Yourcenar, il s'agit essentiellement de mettre en place l'adoption symbolique par la mère de son choix, et l'identification avec elle). Ainsi Yourcenar procède à la modification du statut des personnages n'ayant pas appartenu à la famille auxquels elle souhaite pourtant accorder un rôle symbolique important, ou bien les traiter comme supports de la projection de son identité. Si pour ce faire, elle crée un récit prétendant à la référentialité, sa démarche s'explique par le statut particulier des actes illocutoires accomplis par les textes fictionnels. Dans Qu'est-ce qu'un genre littéraire?, Jean-Marie Schaeffer analyse les noms de genres en fonction de l'acte illocutoire auquel ils se réfèrent, et remarque :

[D]ans la mesure où la plupart des noms qui identifient des pratiques littéraires canoniques se réfèrent à des pratiques discursives d'ordre ludique, ils impliquent une déréalisation des actes illocutoires correspondants, que cette déréalisation procède par mimèsis fictionnelle ou par désancrage communicationnel ${ }^{5}[\ldots]$. (Ibidem : 102-103)

C'est dans ce constat qu'il faut chercher l'explication de la nécessité, pour Yourcenar, d'intégrer le récit fictionnel modifiant la situation familiale à ses chroniques familiales et à sa biographie. Dans Le Labyrinthe du monde, la performativité résulte de la juxtaposition des séquences ou parties génériquement hétérogènes, et surtout dans l'intégration du texte référentiel à l'énonciateur réel et du texte fictionnel ayant le même énonciateur, sans que ce dernier soit marqué comme un fragment à part. Yourcenar souhaite justement minimiser la

\footnotetext{
${ }^{5}$ Nous nous référons au texte de Schaeffer car la notion de déréalisation nous semble plus pertinente que celle de feintise, et plus apte à rendre compte du statut complexe du texte de fiction.
} 
déréalisation des actes illocutoires, pour qu'une déclaration assertive ${ }^{6}$ puisse être prise pour un acte assertif. C'est précisément en instaurant un monde textuel qui s'étend sur plusieurs siècles et plusieurs générations, où les histoires individuelles sont ancrées dans la réalité historique et sociale, et leur authenticité garantie par « la strate de la preuve testimoniale » (Cohn 2001 : 177) qu'elle peut introduire les histoires de Jeanne et d'Egon comme valables et recevables.

Notre dernière remarque concerne la question de la constitution performative du sujet, que nous n'abordons qu'en guise d'ouverture. Lors d'un entretien, l'auteure se penche sur deux conceptions du sujet : selon la première, que l'on trouve chez Proust aussi bien que chez les bouddhistes, c'est « une sorte d'agglomérat, une sorte de paquet de sensations, d'émotions, de souvenirs, qui n'a rien en lui-même de consistant, qui est fait entièrement du reflet des situations, des circonstances, du milieu sur lui »; selon la seconde, représentée par les tragédies grecques ou les tragédies classiques françaises, c'est « un noyau solide représentant une sorte d'entité presque inaltérable qui s'oppose au reste du monde et résiste aux circonstances ». Yourcenar trouve qu'il y a « du vrai dans les deux points de vue, et la plupart des êtres se présentent tour à tour comme cet agglomérat transitoire, et (par une contradiction inexplicable), comme cette espèce de noyau inaltérable et éternel » (Rosbo 1972 : 72).

La trilogie de Yourcenar, comme on l'a constaté dans les textes critiques, est habitée par cette même dualité. Yourcenar se pose comme sujet souverain, maître d'elle-même et de son écriture, qui paradoxalement insiste sur l'impermanence des êtres humains, mais doit raconter les vies des êtres qui se concevaient plutôt comme des noyaux inaltérables, et n'accédaient au décentrement que dans des moments exceptionnels. Dans les deux premiers volumes, il lui suffit de thématiser l'expérience que le sujet fait de sa propre impermanence, lorsque le matériel traité lui en donne l'occasion - même si une telle expérience ne peut jamais être représentée sans être réduite. Dans le dernier volume, Yourcenar, qui repousse et marginalise le récit de sa propre vie et observe que son identité passée lui est désormais lointaine et étrangère, finit par mettre en æuuvre (et non représenter) cette conception du sujet qui sans cesse se fait et se défait, par une écriture devenue performative. Elle se présente authentiquement non dans l'écriture de son autobiographie, qui témoignerait de l'ambition de saisir une version définitive de soi, mais dans le processus d'une écriture fictionnalisée de l'autre, qui devient un acte d'autoposition sans cesse différé et renouvelé. Et Le Labyrinthe du monde, conçu comme chronique/autobiographie, se construit ainsi en texte performatif.

\footnotetext{
6 Dans sa définition des déclarations assertives, Searle insiste sur la présence d'une autorité compétente pour statuer sur les faits. Justement, Yourcenar s'érige en autorité dans son double statut de fille et d'écrivaine : elle s'impose en représentante et de l'institution familiale et de l'institution littéraire.
} 


\section{Références bibliographiques}

\section{Sources}

Yourcenar 1982 : M. Yourcenar, Euvres romanesques, Paris : Gallimard.

Yourcenar 1991 : M. Yourcenar, Essais et mémoires, Paris : Gallimard.

Yourcenar 1997 : M. Yourcenar, Les yeux ouverts : entretiens avec Matthieu Galey, Paris : Bayard Éditions.

Rosbo 1972 : P. de Rosbo, Entretiens radiophoniques avec Marguerite Yourcenar, Paris : Mercure de France.

\section{Références bibliographiques}

Austin 1970 : J. L. Austen, Quand dire, c'est faire, traduit de l'anglais par Gilles Lane, Paris : Éditions du Seuil.

Cohn 2001 : D. Cohn, Le propre de la fiction, traduit de l'anglais (États-Unis) par Claude Hary-Schaeffer, Paris : Éditions du Seuil.

Derrida 1990 : J. Derida, "Signature événement contexte », in Limited Inc, Paris : Galilée, $17-51$.

Felman 1980 : S. Felman, Le scandale du corps parlant: Don Juan avec Austin ou la séduction en deux langues, Paris : Éditions du Seuil.

Fish 1980 : S. Fish, "How To Do Things with Austin and Searle: Speech-Act Theory and Literary Criticism", in Is there a text in this class ?: the authority of interpretive communities, Cambridge, Mass. ; London : Harvard University Press, 197-245.

Gaudin 1994 : C. Gaudin, Marguerite Yourcenar à la surface du temps, Amsterdam : Rodopi.

Genette 2004 : G. Genette, Fiction et diction, précédé de Introduction à l'architexte, Paris : Éditions du Seuil.

Harris 1995 : N. Harris, « Le Labyrinthe du monde : roman historico-didactique », in Roman, histoire et mythe dans l'œuvre de Marguerite Yourcenar, Tours : SIEY, 229-238.

Lejeune 1996 : P. Lejeune, Le pacte autobiographique, Paris : Éditions du Seuil.

Maindron 1988 : A. Maindron, «L'être que j'appelle moi... », in Marguerite Yourcenar, biographie, autobiographie, València : Universitat de València, 169-176.

Miller 2001 : J. H. Miller, Speech acts in literature, Stanford, California : Stanford University Press.

Miller 2005 : J. H. Miller, Literature as conduct: speech acts in Henry James, New York: Fordham University Press.

Ohmann 1971 : R. Ohmann, "Speech Acts and the Definition of Literature", in Philosophy \& rhetoric, Vol. 4, No. 1, University Park : Pennsylvania State University Press, 1-19.

Ortiz Robles 2010 : Ortiz Robles, The novel as event, Ann Arbor : University of Michigan Press.

Park 2003 : S. A. Park, La Fonction du lecteur dans Le Labyrinthe du monde de Marguerite Yourcenar, Paris : L'Harmattan. 
Peternai 2005 : K. Peternai, Učinci književnosti: performativna koncepcija pripovjednog teksta, Zagreb : Disput.

Petrey 1990 : S. Petrey, Speech Acts and Literary Theory, New York, London : Routledge. Proust 2001 : S. Proust, Quoi ? L'Éternité de Marguerite Yourcenar, Paris : Gallimard.

Savigneau 1990 : J. Savigneau, Marguerite Yourcenar, l'invention d'une vie, Paris : Gallimard.

Schaeffer 1989 : J.-M. Schaeffer, Qu'est-ce qu'un genre littéraire ?, Paris : Éditions du Seuil.

Searle 1982 : J. R. Searle, Sens et expression : études de théorie des actes de langage, traduction par Joëlle Proust, Paris : Les Éditions de Minuit.

Van der Starre 1995 : E. Van der Starre, « Entre roman et histoire », in Roman, histoire et mythe dans l'œuvre de Marguerite Yourcenar, Tours : SIEY, 419-429.

Site internet

$<$ http://www.gallimard.fr/Catalogue/GALLIMARD/Blanche>. 07/11/2018.

\section{Nađa Đurić}

\section{Lavirint sveta Margerit Jursenar kao performativni tekst}

Rad se poziva na Ostinovu i Serlovu teoriju govornih činova i njenu primenu u književnoj teoriji i kritici kako bi se objasnila specifičnost trotomne porodične trilogije Margerit Jursenar Lavirint sveta (Pobožna sećanja, 1974; Arhive sa severa, 1977; Šta? Večnost, 1988, posthumno). Ispituje se žanrovska neodređenost trilogije, koja obuhvata žanrove kao što su porodična hronika, autobiografija, biografija i roman. Kratak osvrt na raznovrsne koncepcije performativnosti fikcionalnog teksta priprema analizu žanrovske složenosti i brisanja granice između dokumentarnog i fikcionalnog u Lavirintu sveta, kao načina uspostavljanja performativne moći teksta. Ovakav postupak povezuje se sa voljom Jursenarove da željenu verziju svoje porodične istorije integriše u stvarnost, pri čemu se sam čin pisanja ukazuje kao čin konstituisanja subjekta.

Ključne reči: Margerit Jursenar, Lavirint sveta, performativ, govorni činovi, žanr, fikcija. 